\title{
Neue und einheitliche Schuldenbegrenzungsregeln für Bund und Länder? - Notwendigkeit und Grenzen der Länderautonomie in der Verschuldungspolitik
}

\section{Art. 115 GG-Schuldenbremse ohne Bremser}

Die Ende 2006 vollmundig als zweite Stufe der Föderalismusreform ${ }^{1}$ angekündigte Neuordnung der Finanzbeziehungen von Bund und Ländern scheint im Frühjahr 2008 ins Stocken geraten zu sein. ${ }^{2}$ Schon zuvor, unmittelbar nach Beginn der Beratungen der Föderalismuskommission II, hatte sich ihr Themenbereich verengt. ${ }^{3}$ Einigkeit zwischen Bund und Ländern besteht zur Zeit offenbar nur darin, dass die ausufernde Staatsverschuldung eingedämmt werden muss und es hierzu neuer (Verfassungs-) Rechtsnormen bedürfe. Auch hier scheint aber der Reformeifer nachzulassen; die Politik steht unter dem Eindruck, dass in unmittelbarer Zukunft die Konjunktur erlahmen, die Staatsausgaben steigen und die zuletzt sprudelnden Steuereinnahmen wieder sinken könnten. An die weiteren und systematisch der Verschuldung vorgelagerten Teilthemen des föderalistischen Finanzsystems - Ausgabenverteilung, Steuerautonomie und Finanzausgleich, also die Regelungsgegenstände der Art. 104a bis 107 GG hat sich von vornherein niemand herangetraut. Wie kommt es dazu, dass ausgerechnet die Begrenzung des Staatskredits im Mittelpunkt aller aktuellen Bemühungen zur Reform der Bund-Länder-Finanzbeziehungen steht?

Staatsverschuldung ist kein originär föderalistisches Thema. $\mathrm{Zu}$ diesem wird es aber, weil erstens zum Schuldenstand des Gesamtstaates von mehr als 1.500 Mrd. Euro (Ende 2007) alle Gebietskörperschaften beigetragen haben. Bund und Länder sind dabei im gegenwärtigen System der vertikalen Zuständigkeits- und Ausgabenverteilung in unterschiedlicher Weise auf Kredite als Einnahmequelle angewiesen. Der Schuldensockel der Länder ist bis hin zu Verschuldungskrisen sehr unterschiedlich. Zweitens: Europäische Vorgaben erfordern die innerstaatliche Koordination der Verschuldungspolitik. ${ }^{4}$ Diese föderalistischen Verknüpfungen der Staatsverschuldung

1 Ob die erste Reformstufe, seit dem 1. September 2006 geltendes Verfassungsrecht (52. Gesetz zur Änderung des Grundgesetzes vom 28. August 2006, BGBl. I 2034), diese Bezeichnung verdient, muss angesichts ihrer begrenzten und wenig überzeugenden Ergebnisse bezweifelt werden, vgl. Peter Selmer, Die Föderalismusreform - Eine Modernisierung der bundesstaatlichen Ordnung?, JuS 2006, 1052 ff.; Stefan Korioth, Neuordnung der Bund-Länder-Finanzbeziehungen, ZG 2007, 1 (6 - 9).

2 Vgl. FAZ v. 26. Februar 2008, S. 10: »Nun geht es ums Geld«.

3 So bereits die Einschätzung von Selmer, Zur Reform der bundesstaatlichen Finanzverfassung, NVwZ 2007, 872 (878).

4 Ein weiterer Grund, warum Staatsverschuldung gerade im Bundesstaat besondere Probleme aufwerfen könnte, nämlich eine (Mit-)Haftung der Gebietskörperschaften, insbesondere des Bundes, für die Verschuldung anderer Gebietskörperschaften, hat mit dem Berlin-Urteil des Bundesverfassungsgerichts (BVerfGE 116, 327) an Brisanz verloren. Das BVerfG anerkennt nunmehr allein in dem Fall, dass ein Land in einen bundesstaatlichen Notstand gera- 
werfen erhebliche Schwierigkeiten auf. Sie umfassen ganz unterschiedliche finanzpolitische Interessen von Bund und Ländern, aber auch der Länder untereinander, die es in der Reform zu bündeln gilt. Man könnte auch sagen: Während der Föderalstaat den Gang in den Schuldenstaat vereinfacht, jedenfalls dann, wenn beiden Ebenen Verschuldungskompetenzen zukommen ${ }^{5}$, erschwert er die Umkehr. Wie kann, so lautet die zentrale Frage, unter den Bedingungen föderaler Vielfalt diese angesichts der Gesamtverschuldung von 1.500 Mrd. Euro dringend erforderliche Umkehr erreicht werden? Erfasst man dies mit dem Begriffspaar Selbständigkeit und Verantwortung, so steht letztere ganz im Vordergrund. Zumindest verlangen die Konsolidierung und die Umkehr aus dem Verschuldungsstaat im gesteigerten Maße das Zusammenwirken von Bund und Ländern. Die Haushaltswirtschaften von Bund und Ländern bewegen sich nicht in voneinander getrennten Räumen. Es gibt zwischen ihnen vielmehr Verklammerungen und Wechselbeziehungen, die sich aus dem einheitlichen Finanzsystem ergeben, das von der einheitlichen Währung über das Steuerrecht bis hin zu einer Einnahmenverteilung zwischen Bund und Ländern reicht, die allen Gebieterkörperschaften eine aufgabengerechte Finanzausstattung verschaffen muss. Der Zugriff beider Ebenen auf den Kapitalmarkt schließlich fordert die Koordinierung der Zugriffsrechte.

Die verfassungspolitische Staatsschuldendebatte zerfällt in zwei Aspekte, die nicht notwendigerweise verschränkt sein müssen. Sie finden zudem in ganz unterschiedlichem Grad Aufmerksamkeit. Im Vordergrund steht die Suche nach den inhaltlichen Kriterien, mit denen in Zukunft die Neuverschuldung von Bund und Ländern durch einschlägige Verfassungsbestimmungen begrenzt und der Abbau der aufgelaufenen Verschuldung in Angriff genommen werden soll. Die verschiedenen Limitierungsmodelle und Empfehlungen lassen sich kaum mehr überblicken. ${ }^{6}$ Die Bundesregierung hat Ende Februar 2008 ein im Bundesministerium der Finanzen entwickeltes Modell in die Kommissionsberatungen eingebracht, das sich am Grundkonzept des Europäischen Stabilitäts- und Wachstumspakts orientiert und dem Ziel eines über einen Konjunkturzyklus annähernd ausgeglichenen Haushalts Rechnung tragen soll; es kombiniert eine »strukturelle Verschuldenskomponente« (jährlich maximal 0,5 des Bruttoinlandprodukts) mit einer konjunkturellen Verschuldenskomponente. Die dar-

ten ist und deshalb seine verfassungsrechtlich notwendigen Aufgaben nicht mehr erfüllen kann, einen Anspruch auf Sanierungshilfen gegen den Bund. Im praktischen Ergebnis ist damit die Hauhaltsnotlage als Anspruchsgrundlage auf Finanzhilfen verabschiedet; der so definierte Notstand dürfte kaum einmal eintreten, ein Haftungsverbund besteht in der Konstellation Haushaltsnotlage nicht (mehr). Problematisch ist daher die Einschätzung des Berlin-Urteils bei Konrad, Vorschläge zur wirksamen Verschuldungsbegrenzung der Länder, Wirtschaftsdienst 2007, 581 (581).

5 Dazu Grossekettler, Für einen Föderalismus mit zentralisierten Verschuldungskompetenzen, in: Konrad / Jochimsen (Hrsg.), Der Föderalstaat nach dem Berlin-Urteil, 2007, 89 (90-92).

6 Knapper Überblick bei Scheller, Zu den langfristigen Implikationen von Verschuldungsverboten, in: Junkernheinrich / Scheller / Woisin (Hrsg.), Zwischen Reformidee und Funktionsanspruch. Konzeptionen und Positionen zur deutschen Finanzverfassung, 2007, 67 (71-72). 
aus abzuleitenden Regeln sollen Bund und Länder verpflichten. Der Bund soll sich strukturell in Höhe von 0,35 des jährlichen Bruttoinlandsprodukts verschulden dürfen (das entspricht derzeit etwa 8,5 Mrd. Euro jährlich), der Rest von 0,15 bliebe der Ländergesamtheit. ${ }^{7}$ In diesem Modell ist der Zwang zur Zentralisierung der Verschuldenskompetenzen unverkennbar.

Der andere Aspekt kreist um die Frage, auf welchem verfahrensrechtlichen Weg eine Einheitlichkeit der Begrenzungsregeln in sechzehn Landesverfassungen und im Bundesrecht im Reformfall erreicht werden kann. Um diesen bislang wenig beachteten Aspekt soll es im Folgenden vorrangig gehen. Weil die Schritte zur Verankerung neuer einheitlicher Kreditgrenzen allerdings nicht völlig von ihrer inhaltlichen Ausgestaltung getrennt werden können, seien einige Anmerkungen zur gegenwärtigen Kreditbegrenzungsdiskussion vorausgeschickt. Ausgeklammert bleibt dabei der ebenso radikale wie diskussionswürdige Vorschlag, die Verschuldungskompetenzen beim Bund zu konzentrieren, dies im Finanzausgleich mit einem System garantierter vertikaler Steuerzuweisungen an die Länder zu verbinden und deren Altschulden auf den Bund zu übertragen. ${ }^{8}$ Dieses Modell, das dann mit dem Bundesstaatsprinzip des Art. 20 Abs. 1 GG vereinbar wäre, wenn den Ländern zugleich ein angemessener Anteil am Gesamtsteueraufkommen im Bundesstaat unentziehbar garantiert ist ${ }^{9}$, hat derzeit keinerlei Aussicht auf Verwirklichung. Deshalb gehen die folgenden Überlegungen davon aus, dass den Ländern - in welcher Weise auch immer - Verschuldungskompetenzen erhalten bleiben.

Heute findet fast ausnahmslos die Auffassung Zustimmung, wonach das Grundkonzept einer nachfrageorientierten diskretionären Fiskalpolitik, das auch Art. 115 Abs. 1 GG und den entsprechenden Normierungen der Landesverfassungen zugrunde liegt, kein taugliches Programm der Schuldenbegrenzung formulieren könne und als Rechtsregel versagt habe. An der »Revisionsbedürftigkeit der geltenden verfassungsrechtlichen Regelungen« sei, so meint auch das Bundesverfassungsgericht, »kaum noch zu zweifeln ${ }^{10}$. Aber das erweist sich schnell als fragwürdig. ${ }^{11}$ Das Problem liegt darin,

7 Kommissionsdrucksache 096 vom 28. Februar 2008. Dazu Groneck/Plachta, Close to balance oder Nettoinvestitionen? - Kreditgrenzen für die Bundesländer, Wirtschaftsdienst 2008, S. 115 ff.; Blankart / Fasten, Föderalismusreform auf der Kippe, FAZ v. 29. März 2008, S. 13.

8 Grossekettler (Fn. 5), 89 ff.; Halsch, Entmachtet und entschuldet die Länder, Die Zeit v. 05. Oktober 2006, S. 38.

$9 \mathrm{Zu}$ dieser Grundanforderung an jedes bundesstaatliche Verteilungssystem bereits BVerfGE $34,9(20)$.

10 BVerfG, Urt. vom 9. Juli 2007 (2 BvF 1/04), Absatz-Nr. 133. In diesem Sinne jetzt auch die »Berliner Erklärung « der Konferenz der Präsidentinnen und Präsidenten der deutschen Landesparlamente vom 31. August 2007 (Kommission von Bundestag und Bundesrat zur Modernisierung der Bund-Länder-Finanzbeziehungen, Drucksache 048), S. 1: »Das bestehende Regelwerk zur Beschränkung der staatlichen Kreditaufnahme hat den fortwährenden Anstieg der Verschuldung in Bund und Ländern nicht aufhalten können.«

11 Die Verwirrung zeigt eine merkwürdige Formulierung von Gaitanides, Der Nationale Stabilitätspakt nach der Föderalismusreform - eine Fiktion?, NJW 2007, 3112 (3113): »Die Vorgaben der Verfassung führen also faktisch (!) zu einer Haushaltspolitik der Länder, der rechtlich (!) keine Verschuldungsgrenzen gesetzt sind.« 
dass die Haushaltsgesetzgeber der letzten 40 Jahre Art. 115 Abs. 1 GG weniger angewendet als umgangen haben. Statt der Norm des Art. 115 Abs. 1 GG hat in der Haushaltspolitik gegolten, was diese Norm gerade untersagt. Vor allem ist die Vorschrift als ständige Ermächtigung und Erlaubnis zur Nettokreditaufnahme bis zur Höhe der gleichzeitig getätigten Investitionen (miss)verstanden worden, obwohl sie bei richtigem Verständnis ein grundsätzliches Verbot der Verschuldung in der Normallage ausspricht. Das Wort Regelkreditobergrenze hat das Verschwinden des Grundgebots, Verschuldung zu vermeiden, sinnfällig zum Ausdruck gebracht. Geflissentlich übersehen wurde dabei der bereits vom verfassungsändernden Gesetzgeber herausgestellte Zusammenhang des Art. 115 Abs. 1 GG mit dem Gebot des Art. 109 Abs. 2 GG, wonach die Haushaltswirtschaft von Bund und Ländern den Erfordernissen des gesamtwirtschaftlichen Gleichgewichts Rechnung zu tragen hat. ${ }^{12}$ Das Bundesverfassungsgericht hat kürzlich daran erinnert: »auch für die Kreditaufnahme« sind »die konjunkturpolitischen Vorgaben im Sinne von Art. 109 Abs. 2 GG [...] maßgeblich «. ${ }^{13}$ Daraus sollte nach der Vorstellung des Jahres 1969 keineswegs folgen, dass der Haushaltsgesetzgeber auch in konjunkturellen Gleichgewichts- oder Boomphasen bis zur Höhe der Investitionen Kredite aufnehmen dürfe. Diese Obergrenze darf vielmehr nur genutzt werden, soweit dies mit den Forderungen des gesamtwirtschaftlichen Gleichgewichts vereinbar ist. Im Jahre 1989 hat das Bundesverfassungsgericht betont, Art. 109 Abs. 2 GG solle auch vor und außerhalb einer Störung des gesamtwirtschaftlichen Gleichgewichts eine Limitierungsfunktion für die staatliche Kreditaufnahme wahrnehmen. Art. 109 Abs. 2 GG soll »u. a. verhindern, dass sich - jeweils unterhalb der Höchstgrenze des Art. 115 Abs. 1 S. 2 Halbs. 1 GG - ein stetig wachsender Schuldensockel bildet, der schließlich die Fähigkeit des Staatshaushalts, auf die Probleme der Gegenwart und der Zukunft zu reagieren, in Frage stellt. « ${ }^{14}$ Mit anderen Worten: Das geltende Recht verbietet prozyklische Verschuldung. »Hiernach kann beispielsweise die Kreditaufnahme gering zu halten oder eine im gesamtwirtschaftlichen Interesse eingegangene erhebliche Verschuldung zurückzuführen sein, falls ein solches Haushaltsgebaren im Hinblick auf eine an der Erhaltung des gesamtwirtschaftlichen Gleichgewichts ausgerichtete mittelfristige Wirtschaftspolitik vonnöten erscheint. « ${ }^{15}$ Das hat die Politik, wie auch andere Mahnungen und Aufforderungen aus dem Staatsverschuldungs-Urteil des Jahres 1989, souverän in den Wind geschlagen. Wenn heute konstatiert wird, Art. 115 GG habe den Gang in den Schuldenstaat nicht verhindert, und - unter Anspielung auf Verfassungsänderungen - die zweite große Koalition müsse nun im Schuldenrecht auslöffeln, was die erste eingebrockt habe ${ }^{16}$, dann

12 Vgl. BT-Drs. V/3040, S. 39 (Tz. 60); BVerfGE 79, 311 (330 f.)

13 BVerfG, Urt. vom 9. Juli 2007 (2 BvF 1/04), Absatz-Nr. 123.

14 BVerfGE 79, 311 (355 f.)

15 BVerfGE 79, 311 (334). Daran erinnert auch Höfling, Haushalts- und Finanzverfassung in der Krise, Der Staat 46 (2007), 163 (166): »Die finanzpolitischen Akteure dürfen deshalb die Investitionssummengrenze nicht regelmäßig ,ausreizen', sondern müssen sich auch in einer mittelfristigen Perspektive ihre intertemporale Stabilisierungsfähigkeit erhalten.«

$16 \mathrm{Vgl}$. Schäfers, Rein in den Schuldenstaat, raus aus dem Schuldenstaat, FAZ v. 14. September 2007, S. 14. 
schwingt dabei eine problematische Ursachen-Wirkung-Behauptung mit. Ob das Konzept des Art. 115 Abs. 1 GG von Anfang an verfehlt war, übermäßige Verschuldung also ermöglicht oder begünstigt hat, ist keineswegs ausgemacht. Denn: Das Konzept kam entgegen den verfassungsrechtlichen Anforderungen in der Politik nicht zur Entfaltung. Interessant ist deshalb die Frage, die leider hypothetisch bleiben muss, ob ein anderes Verschuldungskonzept den Gang in den Schuldenstaat verhindert hätte. Daran bestehen erhebliche Zweifel. Die Hauptgründe der Verschuldung waren die Ausweitung sozialer Leistungen bis zum Beginn der 1980er Jahre und die deutsche Einigung. Etwa 70 v. H. der Verschuldung des öffentlichen Gesamthaushalts ist nach 1990 entstanden. ${ }^{17}$ In beiden Bereichen hat die Politik Leistungsansprüchen und Erwartungen der Bevölkerung entsprochen. Angesichts der in diesen Situationen festzustellenden Steuerungsschwäche des Staatsschuldenrechts hätte jede Limitierungsregel, vom wenig sinnvollen absoluten Neuverschuldungsverbot abgesehen, kaum wirksame Dämme errichten können. Keine Verschuldensregel kann so strikt sein, dass sie wie auch immer begründete Ausnahmefälle von vornherein ausschließen könnte. ${ }^{18}$

Was ist daraus für die Reformaufgabe in der zweiten Stufe der Föderalismusreform zu lernen? Ob es ökonomisch überzeugendere Verschuldungsregeln als die des geltenden Rechts gibt, ist nicht der entscheidende Punkt, denn ob diese im Fall einer Aufnahme in das Verfassungsrecht mehr bewirken als das geltende Recht, steht keineswegs fest. Ausschlaggebend ist das Gelingen eines finanz- und haushaltspolitischen Mentalitätswechsels, der darauf gerichtet sein muss, die vorhandene Kreditlimitierungsregel - wie auch immer sie inhaltlich gestaltet ist - ernst zu nehmen. Im Sondervotum zum Urteil des Bundesverfassungsgerichts vom 9. Juli 2007 heißt es dazu treffend: »Eine unkontrollierte Talfahrt öffentlicher Finanz- und Haushaltswirtschaft kann nicht so sehr durch rechtspolitische Forderungen nach besseren Bremsen verlangsamt werden, sondern zuallererst durch die Betätigung der bereits vorhandenen Bremsen. ${ }^{19}$ Auch im Haushaltsrecht muss sich die Vorstellung durchsetzen, dass die Verfassung »die Finanzmacht festen Regeln unterwirft ${ }^{20}$, die abweichenden Handhabungen und Kompromissen nicht zugänglich ist. ${ }^{21}$ Damit dies gelingt, hat eine reformierte Schuldenregel darüber hinaus einfach und klar auszufallen - jede komplizierte, von ökonomischen Wertungen abhängige Regel verringert die Chance, Umdeutungen und Umgehungen zu vermeiden. Auch unter diesem Aspekt würde es völlig genügen, die Einbruchstellen der Steuerungsschwäche in das geltende Recht zu schließen, indem

17 Vgl. Lenk/Kuntze, Der Einfluss der Deutschen Vereinigung auf die Staatsverschuldung, in: Junkernheinrich / Scheller / Woisin (Fn. 6), 21 (21 ff.); Gase, Die Neuordnung der BundLänder-Finanzbeziehungen - Perspektiven und Positionen der ostdeutschen Länder, in: Junkernheinrich / Scheller / Woisin (Hrsg.), (Fn. 6), 41 (42).

18 Dazu Kerber, Überschuldete Bundesländer: Ist die Einführung eindeutiger Verschuldungsgrenzen sinnvoll?, ifo-Schnelldienst 2/2007, 17 (17).

19 BVerfG, Urt. vom 9. Juli 2007 (2 BvF 1/04), Sondervotum, Absatz-Nr. 162.

20 Vgl. Isensee, Budgetrecht des Parlaments zwischen Schein und Sein, JZ 2005, 971 (971).

21 Zum Finanzausgleich BVerfGE 72, 330 (389): Das Grundgesetz habe auch in diesem Bereich »rechtliche Positionen, Verfahrensregeln und Handlungsrahmen festgelegt, die Verbindlichkeit beanspruchen.« 
vor allem der einfachgesetzliche Investitionsbegriff durchgreifend verengt und die Handlungsmöglichkeiten nach Art. 109 Abs. 4 GG aktiviert würden. Auch sollte der Zwang zur Schuldentilgung gesetzlich geregelt werden. Bei allem ist nicht theoretische Brillanz gefordert, sondern ein einfaches, transparentes und kontrollierbares Regelwerk. Schließlich gibt noch ein weiterer Befund zu denken: Die in den 1960er Jahren eingeführten Regeln fanden damals den Beifall, heute die scharfe Kritik der Ökonomie. Wer garantiert, dass es einer reformierten Schuldenregel anders ergehen wird, dass sie also in 20 Jahren nicht zum Spott der Ökonomen wird? Hier ist daran zu erinnern, dass das Verfassungsrecht nicht dazu da ist, dem Wandel unterworfene Thesen und Lehren anderer Disziplinen zu verrechtlichen. Die Zeitbedingtheit jeder Norm, die jeweils aktuelle wirtschaftswissenschaftliche Konzepte in den »Aggregatzustand des Rechts « ${ }^{22}$ überführt, gerät in Konflikt mit der Aufgabe der Verfassung, eine möglichst dauerhafte, Moden und wechselnden Anschauungen entzogene rechtliche Grundordnung zu schaffen.

\section{Handlungsspielräume und Verfahrenswege zur Reform der Verschuldungsregeln in Bund und Ländern}

Sollte sich der verfassungsändernde Gesetzgeber im Zuge der Föderalismusreform II nach dem bereits bei der Reformstufe I geltenden Leitprinzip, dass sich etwas ändern müsse - dennoch zur Novellierung der Verschuldungsregeln entschließen, so sind seine Handlungsspielräume im Lichte des europäischen Gemeinschaftsrechts und der Garantie des Bundesstaatsprinzips (Art. 20 Abs. 1, 79 Abs. 3 GG) sowie die innerstaatlichen Verfahrenswege auszuloten, mit denen neue Regeln Verbindlichkeit für die Haushaltsgesetzgeber im Bund und Ländern erlangen könnten.

\section{Vorgaben des Art. 104 EGV}

Das Europäische Gemeinschaftsrecht enthält im Ergebnis, trotz seines Anwendungsvorrangs gegenüber allem nationalen Recht und trotz des Gebots gemeinschaftsrechtskonformer Auslegung des nationalen Rechts, wenig Restriktionen für den Entscheidungsbereich des verfassungsändernden Gesetzgebers. Art. 104 EGV verpflichtet die Mitgliedstaaten, übermäßige öffentliche Defizite zu vermeiden. ${ }^{23}$ Verstöße gegen die Haushaltsdisziplin setzen ein Verfahren in Gang, das letzten Endes zu Sanktionen gegen den Mitgliedstaat führen kann. ${ }^{24}$ Nach Art. 3 des Protokolls über das Verfahren bei einem übermäßigen Defizit haben die Mitgliedstaaten überdies zu gewährleisten, »dass die innerstaatlichen Verfahren im Haushaltsbereich sie in die Lage versetzen,

22 Vgl. Isensee, Schuldenbarrieren für Legislative und Exekutive, in: Rudolf Wendt (Hrsg.), Staat, Wirtschaft, Steuern. Festschrift für Karl Heinrich Friauf, 1996, 705 (708).

23 Dazu Häde, in: Calliess / Ruffert (Hrsg.), EU/EG, 3. Auflage 2007, Art. 104 EGV; Konow, Der Stabilitäts- und Wachstumspakt, 2002.

24 Die bislang geringe Effektivität und politisch gesteuerte Anwendung des europäischen Regelwerkes bestätigt, dass es bei Schuldenbremsen auf den Willen zur Betätigung ankommt. 
ihre sich aus diesem Vertrag ergebenden Verpflichtungen in diesem Bereich zu erfüllen «. Diese speziell für bundesstaatlich organisierte Gemeinschaftsmitglieder geschaffene Vorschrift gibt verbindlich jedoch lediglich das Ziel vor. Es fehlen verbindliche Verfahrensregelungen und erst recht werden den mitgliedstaatlichen Zentralregierungen keine innerstaatlichen Befugnisse verliehen. Diese zu schaffen, ist ausschließlich Sache des nationalen Rechts. Wie die Mitgliedstaaten ihre Verpflichtung erfüllen, bleibt ihnen überlassen. ${ }^{25}$ Daran hat auch der 2006 in das Grundgesetz eingefügte Art. 109 Abs. 5 nichts geändert, nach dessen Satz $1 »$ Verpflichtungen der Bundesrepublik Deutschland aus Rechtsakten der Europäischen Gemeinschaft auf Grund des Artikels 104 des Vertrags zur Gründung der Europäischen Gemeinschaft zur Einhaltung der Haushaltsdisziplin [...] von Bund und Ländern gemeinsam zu erfüllen « sind. Diese Vorschrift enthält keine komplette Transformation der gemeinschaftsrechtlichen Vorgaben in das Grundgesetz mit der Folge, dass jetzt die 17 Haushaltsgesetzgeber jeweils unabhängig voneinander die Defizit-Kriterien des »Stabilitäts- und Wachstumspakts « einzuhalten hätten. ${ }^{26}$ Gemeint ist nur die Haftung für Sanktionen ${ }^{27}$, ansonsten steht das nationale Verschuldungsregime selbständig neben den Vorgaben des europäischen Rechts.

\section{Der Verfassungsrahmen der Art. 20 Abs. 1, 109 Abs. 1 GG}

Auch unabhängig von europäischer Vorbelastung ist es sinnvoll, den zurzeit bestehenden weitgehenden Gleichlauf der Verschuldungsregelungen im Grundgesetz und in den Landesverfassungen beizubehalten. Je nach inhaltlichem Regelungskonzept kann es jedoch auch Gründe für Unterschiede auf der Bundes- und Landesebene geben. So ist zu beachten, dass bei der gegenwärtigen vertikalen Aufgaben-, Ausgaben- und Einnahmenverteilung die Kreditfinanzierung in den Länderhaushalten teilweise andere Hintergründe als beim Bund hat. Während der Bund über die wesentlichen Befugnisse der Einnahmegesetzgebung verfügt, insbesondere über Steuern, können sich die Länder derzeit nur über eine erhöhte Verschuldung relevante Einnahmespielräume verschaffen. Sehr viel schneller als der Bund geraten die Länder in den Zwang zur Kreditaufnahme, zumal die Aufgaben und Ausgaben der Länder vielfach bundesgesetzlich vorgegeben sind. ${ }^{28}$ Daran hat - mit Ausnahme des Überganges des Besoldungsrechts der Landesbeamten auf die Länder (Art. 74 Abs. 1 Nr. 27 GG) - die Föderalismusreform 2006 nichts geändert. Die seit 1990 im Vergleich der Länderhaushalte auffallend

25 Einschränkend Häde, Europarechtliche Einwirkungen auf die nationale Finanzverfassung, in: Konrad / Jochimsen (Hrsg.), Finanzkrise im Bundesstaat, 2. Auflage 2007, 196 (201 ff.), wonach die finanzverfassungsrechtlichen Vorschriften des Grundgesetzes gemeinschaftsrechtkonform auszulegen sind.

26 So aber Jarass, in: ders. / Pieroth, GG, 9. Auflage 2007, Art. 109 GG Rdnr. 7; ähnlich auch Häde, Europäische Haushaltsdisziplin als Maßstab innerstaatlicher Schuldenschranken, in: Junkernheinrich / Scheller / Woisin (Hrsg.) (Fn. 6), 133 (140 f).

27 Classen, Hauptstadtfrage und Verbesserung der Europatauglichkeit, in: Starck (Hrsg.), Föderalismusreform, 2007, Rdnr. 259.

28 Neuere Untersuchungen vertreten allerdings den Standpunkt, dass dennoch die Variationsbreite der Länder bei solchen Ausgaben durchaus beträchtlich ist. 
gleichförmige Verschuldung (Ausnahmen bilden vor allem Bayern, Berlin und Bremen) deutet finanzielle Zwänge an, die nicht allein auf individuelle landespolitische Entscheidungen zurückführbar sein können. Diese qualitative Verschiedenheit von Bundeshaushalt und Länderhaushalten muss jede Reform beachten.

Unter diesem Aspekt ist der jüngst von der Bundesregierung in die Reformdiskussion eingebrachte Vorschlag problematisch, in Anlehnung an die europäischen Kriterien zur Haushaltsdisziplin die Gesamtverschuldung der Gebietskörperschaften auf 0,5 v. H. des Bruttoinlandsprodukts zu begrenzen und nur 0,15 des Bruttoinlandsprodukts als Orientierungsgröße für die Gesamtverschuldung aller Länder zuzulassen. Auch unter Berücksichtigung der konjunkturellen Elemente, die kurzfristig höhere Verschuldung ermöglichen sollen, brächte das die Länder bei einem Konjunktur- und Steuereinnahmenrückgang in Schwierigkeiten. Dies müsste jedenfalls dann gelten, wenn eine gleichzeitige Verstärkung der Bestimmungsmöglichkeiten der Länder über Steuern und über Leistungsstandards auf der Ausgabenseite unterbliebe. Das Modell der Bundesregierung kann nur dann weiterverfolgt werden, wenn es in eine umfassende Stärkung der Länderautonomie eingebettet wird. Soll es dagegen als eine isolierte, starre oder niedrig gezogene Verschuldensgrenze der Länder verstanden werden, dann steht bei fallenden Steuereinnahmen deren Fähigkeit in Gefahr, auf der Grundlage einer aufgabengerechten Finanzausstattung ihre Aufgaben selbstbestimmt zu erfüllen. Im Extremfall könnte dies die Staatlichkeit der Länder vor eine Zerreißprobe stellen und deshalb im Konflikt mit der Garantie der Essentialia der Bundesstaatlichkeit nach Art. 20 Abs. 1 i.V.m. Art. 79 Abs 3 GG geraten.

Für eine unterschiedliche Behandlung von Bund und Ländern gibt es noch einen weiteren Anwendungsfall. Wenn am Grundkonzept des Art. 115 Abs. 1 GG festgehalten werden sollte und eine erhöhte Verschuldung bei einer Störung des gesamtwirtschaftlichen Gleichgewichts erlaubt bleibt, sollte eine Ausweitung der Neuverschuldung im Fall einer Störung des gesamtwirtschaftlichen Gleichgewichts auf Länderebene ausgeschlossen sein. Es ist wenig sinnvoll, in einem einheitlichen Wirtschaftsgebiet eine auf das Gebiet eines Bundeslandes sich beziehende Störung des gesamtwirtschaftlichen Gleichgewichts als Maßstab einer ausnahmsweise erhöhten Verschuldung anzuerkennen. In der Praxis haben die Erfahrungen der letzten Jahre auch gezeigt, dass eine solche Störung nicht sinnvoll dargelegt werden kann.

Sollten sich die Verschuldungsregeln des Bundes und der Länder qualitativ oder nach der Quantität der jeweiligen zur Verfügung stehenden Verschuldungsanteile auseinanderentwickeln, so muss in jedem Fall die verschiedene Bedeutung der Kreditaufnahme für die beiden Ebenen aufgefangen werden. Je stärker der Bund zukünftig die Bestimmung über die fiskalische Handlungsmöglichkeit Kreditaufnahme unter Verdrängung der Länder erhalten sollte, desto stärker muss sich entweder der Bund in die Pflicht nehmen lassen, für eine aufgabengerechte Finanzausstattung der Länder einzustehen oder die Selbstbestimmung der Länder über Einnahmen und Ausgaben insgesamt verstärkt werden. 


\section{Verfahrenswege zu einheitlichen Verschuldungsgrenzen}

Auf welche Weise kann aber nun erreicht werden, dass eine Reform der Verschuldungsmaßstäbe und -grenzen alle Haushaltsgesetzgeber - mit den gerade geschilderten Maßgaben der vertikalen Differenzierung - bindet? Hierzu kommen mehrere Wege in Betracht, die im Lichte der Garantie des Art. 109 Abs. 1 GG gewürdigt werden müssen, wonach Bund und Länder zur Gewähr ihrer politischen Autonomie ${ }^{29}$ in ihrer Haushaltswirtschaft selbständig und voneinander unabhängig sind. Der geschützte Bereich der Haushaltswirtschaft ist enger als die Finanzwirtschaft von Bund und Ländern ${ }^{30}$, die mannigfaltigen Einschränkungen und gegenseitigen Abhängigkeiten unterliegt. »Die getrennten Haushaltswirtschaften sind notwendig Bestandteil einer übergreifenden Finanzwirtschaft, die durch höherrangiges Recht konstituiert wird. ${ }^{31}$ So bestimmen nach Art. 105 GG die Steuergesetze (praktisch ausschließlich des Bundes) und die Verteilungsregeln der Art. 106, 107 GG über die Einnahmen von Bund und Ländern; Art. 91a und Art. 91b, Art. 104a und 104b GG determinieren über die Ausgabenlasten die Ausgabenseite. Die in Art. 109 Abs. 1 GG geschützte Haushaltswirtschaft bezieht sich daher lediglich auf die haushaltsbezogenen Vorgänge bei den Einnahmen und bei den Ausgaben. Auch hier gibt es indes noch einmal Einschränkungen der eigenen Entscheidungsspielräume, die sich aus anderen Verfassungsnormen ergeben, insbesondere den weiteren Absätzen des Art. 109 GG. ${ }^{32}$ Die Einräumung einer selbständigen und unabhängigen Haushaltswirtschaft prägt die Staatlichkeit des Bundes und der Länder entscheidend mit. Es geht nicht nur um organisatorische Befugnisse, sondern um die Möglichkeit, durch Geldverwendung politische Schwerpunkte zu setzen. Aber die im Grundgesetz selbst, insbesondere in Art. 109 Abs. 2 bis 4 GG, enthaltenen Einschränkungen berühren den Kern der Länderstaatlichkeit (und im Übrigen den der Staatlichkeit des Bundes) nicht. Verfassungsrechtliche Grenzziehungen der Haushaltsautonomie geraten dann nicht in Kollision mit Art. 79 Abs. 3 GG, wenn sie darauf verzichten, gezielte Einflussnahmen auf die Haushaltsführung durch andere Länder oder den Bund zu ermöglichen. Angesichts des an die Spitze des Art. 109 GG gestellten Grundsatzes gilt jedoch, dass für alle Länder und/oder den Bund geltende Einschränkungen ein Verfassungsvorbehalt gilt. Nur Regeln des Grundgesetzes selbst können inhaltliche Beschränkungen der Haushaltsautonomie ermöglichen. Daraus folgt, dass es nicht möglich wäre, die Grundsatzgesetzgebungskompetenz des Bundes nach Art. 109 Abs. 3 GG als Rechtstitel in Anspruch zu nehmen, um für Bund und Länder gemeinsam geltende Verschuldungsregelungen im Haushaltsgrundsätzegesetz oder einem weiteren Grundsatzgesetz aufzustellen. Die Befugnis, Grundsätze für das Haushaltsrecht und eine konjunkturgerechte Haushaltswirtschaft zu regeln, bezieht sich zwar nicht nur auf technische Fragen des Haushalts; soweit es aber um inhaltliche Ziele oder Begrenzungen des Haus-

29 Vgl. BVerfGE 86, 148 (264).

30 Vgl. BVerfGE 101, 158 (220); Siekmann in: Sachs (Hrsg.), GG, 4. Auflage 2007, Art. 109 GG Rdnr. 4 f.

31 Vgl. Rodi, in: Dolzer/ Vogel / Graßhof (Hrsg.), GG, Art. 109 GG Rdnr. 67.

32 Vgl. BVerfGE 1, 117 (131); 20, 56 (94). 
haltsgesetzgebers geht, erlaubt der Kompetenztitel nur die Aufstellung von Grundsätzen. ${ }^{33}$ Substanzielle Bindungen der Haushaltsgesetzgeber in Bund und Ländern durch erschöpfende Gesamtregelungen der erfassten Materien sind dadurch nicht gedeckt. Die gesetzliche Einführung verbindlicher Verschuldungsgrenzen könnte daher nicht auf Art. 109 Abs. 3 GG gestützt werden. ${ }^{34}$ Aus der Rechtsprechung des Bundesverfassungsgerichts folgt nichts anderes. Zwar hat das Gericht im Zusammenhang seiner Finanzausgleichsentscheidung des Jahres 1992 angenommen, der Gesetzgeber könne gestützt auf Art. 109 Abs. 3 GG finanzwirtschaftliche Kennziffern und Grenzen, auch zur Kreditfinanzierung, aufstellen. Es hat in diesem Zusammenhang aber nur von daraus folgenden Obliegenheiten des Bundes und der Länder gesprochen. ${ }^{35}$

Möglich wäre es aber, durch eine Änderung der Art. 115 und 109 GG neue Grenzen der Neuverschuldung in Bund und Ländern aufzustellen. Alle Gebietskörperschaften im Bundesstaat sind verpflichtet, auf die Interessen der anderen Rücksicht zu nehmen. Grenzen der freien Haushaltswirtschaft im Interesse der gesamtstaatlichen Finanzwirtschaft können in Gestalt einer Normativbestimmung gezogen werden; das Grundgesetz kann die Länder durchaus zwingen, finanzbezogene Bestimmungen in ihren Verfassungen nach grundgesetzlichen Vorgaben zu ändern. ${ }^{36}$ Eine noch einmal andere Regelungstechnik kommt zur Anwendung, wenn das Grundgesetz selbst in Gestalt einer Durchgriffsbestimmung ${ }^{37}$ für Bund und Länder unmittelbar geltendes Verfassungsrecht setzt, das dann entgegenstehendes Landesverfassungsrecht, soweit es besteht, verdrängt. Diese Regelungstechnik - die Bestimmungen der Bundesverfassung werden nicht Inhalt der Landesverfassung, sondern gelten neben der Landesverfassung für die Landesstaatsgewalt - entspräche zwar nicht der Selbständigkeit der Verfassungsräume von Bund und Ländern. ${ }^{38}$ Sie kommt darin zum Ausdruck, dass jede staatliche Gebietskörperschaft selbstbestimmt in einer eigenen Verfassung ihr Staatsorganisationsrecht und damit auch ihr Finanz- und Haushaltsverfassungsrecht normiert. Es entspricht weiterhin nicht der ursprünglichen Systematik des Grundgesetzes, das Befugnisse und Bin-

33 Heun, in: Dreier (Hrsg.), GG, 1. Auflage 2000, Art. 109 GG Rdnr. 31.

$34 \mathrm{Vgl}$. Häde, Die innerstaatliche Verteilung gemeinschaftlicher Zahlungspflichten, S. 55; Siekmann (Fn. 30), Rdnr. 29. Auch BVerfGE 116, 327 (393) scheint jetzt für den damit zusammenhängenden Bereich der Prävention von Haushaltsnotlagen dieser Auffassung zuzustimmen, weil das Gericht Lösungskonzepte anmahnt, die »verfassungsrechtlich über Art. 109 Abs. 3 GG hinaus fundiert « sein müssten. Anders Rodi, in: Dolzer/ Vogel / Graßhof (Hrsg.), GG, Art. 109 Rdnr. 607 ff., der die gesetzliche Festlegung von Verschuldungsgrenzen auf der Grundlage des Art. 109 Abs. 3 GG für zulässig hält.

35 Vgl. BVerfGE 86, 148 (266 f).

36 Darauf beruht der Vorschlag des Sachverständigenrates, der in einem neuen Art. 115 Abs. 6 GG sein zunächst für den Bund entwickeltes Konzept der Kreditaufnahme und -tilgung auch den Ländern vorschreiben will, vgl. Sachverständigenrat zur Begutachtung der gesamtwirtschaftlichen Entwicklung, Staatsverschuldung wirksam begrenzen. Eine Expertise im Auftrag des Bundesministers für Wirtschaft und Technologie, 2007, S. 4.

37 Zur Unterscheidung von Normativ- und Durchgriffsnormen Rozek, Das Grundgesetz als Prüfungs- und Entscheidungsmaßstab der Landesverfassungsgerichte, 1993, S. 36 ff.; Nierhaus, in: Sachs (Hrsg.), GG, 4. Auflage 2007, Art. 28 GG Rdnr. 1, 4 f.

38 Vgl. BVerfGE 4, 178 (189); 6, 376 (381 f.); 22, 267 (270); 41, 88 (118); 60, 175 (209); 96, 345 (368 f.); 98, 145 (157). 
dungen des Bundes normiert, nicht aber der Länder, denen pauschal die staatlichen Aufgaben und Befugnisse zugewiesen werden (vgl. Art. 30, Art. 70 ff., Art. 83 ff. GG). Es gibt aber bereits jetzt viele Normen des Grundgesetzes, die in unterschiedlichem Grad die Handlungsmöglichkeiten der Länder begrenzen. Art. 28 Abs. 1 S. 1 GG, wonach die verfassungsmäßige Ordnung in den Ländern bestimmten Staatsstrukturvorgaben des Grundgesetzes entsprechen muss, steht der Bindung der Länder nicht entgegen. Das Grundgesetz kann Verfassungsrecht setzen, das unmittelbar auch für die Landesstaatsgewalt gilt. Solche grundgesetzlichen Kreditgrenzen würden auch die Budgethoheit der Landtage nicht verletzen, denn diese bezieht sich vorrangig auf die Ausgabenseite des Haushalts. Restriktionen der Einnahmen über den Bereich der Abgaben hinaus rechtfertigen sich dadurch, dass - wie im Fall der Besteuerung - auch bei der Kreditaufnahme die Inanspruchnahme der einheitlichen Volkswirtschaft der Koordinierung bedarf. Es sei daran erinnert, dass bereits das geltende Recht in Art. 109 Abs. 4 GG die Möglichkeit eröffnet, zur Abwehr einer Störung des gesamtwirtschaftlichen Gleichgewichts durch zustimmungsbedürftiges Bundesgesetz Höchstbeträge der Kreditaufnahmen der Gebietskörperschaften festzusetzen; von dieser Möglichkeit ist bislang nur in Einzelfällen zu Beginn der 1970er Jahre Gebrauch gemacht worden. ${ }^{39}$ Sollte eine Verfassungsänderung ökonomisch anders begründete Limitierungen der Kreditaufnahme einführen, so hätten es die Länder schließlich in der Hand, durch ihre Mitwirkungen an Grundgesetzänderungen (Art. 79 GG) verfassungsrechtlich bindende Kreditgrenze mitzugestalten. Eine bedeutsame Konsequenz hätten Durchgriffsnormen des Grundgesetzes allerdings: Die Landesverfassungsgerichte dürften einfaches Landesrecht - im konkreten Fall also das Landeshaushaltsgesetz - nicht anhand der grundgesetzlichen Durchgriffsnorm überprüfen, zumindest fehlt ihnen die Verwerfungskompetenz.

Ein weiterer, selbstverständlich möglicher Weg, zu übereinstimmenden Regelungen zu gelangen, bedarf nur kurzer Erwähnung: Bund und Länder fügen in ihre Verfassungen jeweils gleichlautende Regelungen ein, oder, falls vertikal differenziert werden soll, geben sich die Länder gleichlautende Regelungen. Da es aber mehr als unsicher ist, ob es gelingen kann, einen Gleichlauf von 17 (oder 16) Verfassungen zu erreichen, wird neuerdings ein multilateraler Staatsvertrag aller 16 Länder erwogen, mit dem eine Einigung auf einheitliche Kreditgrenzen erreicht werden soll. ${ }^{40}$ Die Verfassungsordnung zieht dem Regelungsbereich eines solchen Vertrages allerdings enge Grenzen. ${ }^{41}$ Zwar können die Landesregierungen (oder, je nach Landesrecht, die Minister-

39 Vgl. Patzig, Konjunktursteuerung im Bundesstaat, DVBl. 1977, 841 (843 ff).

40 Darauf spielt wohl auch die »Berliner Erklärung « der Landtagspräsidenten (Fn. 3), S. 3, an: »Die Eigenstaatlichkeit der Länder schließt nicht aus, dass zur Bewältigung bestehender Haushaltskrisen konjunktureller oder struktureller Art Regelungen getroffen werden, mit denen Bund und Länder sich verpflichten, ihre Haushalte mittelfristig zum Ausgleich zu bringen und ihre Schulden aufgrund von längerfristigen Tilgungsplänen zu reduzieren.«

41 Bereits im Vorfeld der Finanzreform 1967/69 war - unter anderem im die Reform vorbereitenden Gutachten der Troeger-Kommission von 1966 - die Möglichkeit koordinierter Kreditpolitik durch Staatsverträge erwogen, dann aber verworfen worden, weil die Zuständigkeitsordnung von Bund und Ländern durch solche Verträge nicht geändert werden kann. Zu den Diskussionen der 1960er Jahre vgl. Rodi (Fn. 34), Art. 109 Rdnr. 25 ff. 
präsidenten) als Vertreter der Länder Verträge über Materien schließen, die in die Verbandszuständigkeit der Länder fallen. Der Staatsvertrag ist ein seit langem geläufiges Instrument der Länderkooperation. Die vertragsschließenden Regierungen sind dabei aber an ihre jeweiligen Verfassungen gebunden. Ausgeschlossen ist eine vertragliche Verfügung über die Haushaltsautonomie. Wenn der Vertrag darauf abzielen soll, bestehende Verschuldungsgrenzen durch neue zu ersetzen, dann kann er nur die Selbstbindung der Landesregierung enthalten, auf entsprechende Regelungen in den Landesverfassungen durch Initiativen zu Verfassungsänderungen hinzuwirken. Der Vertrag selbst kann selbstverständlich kein Verfassungsrecht setzen, dazu bedarf es der parlamentarischen Gesetzgebung mit Zwei-Drittel-Mehrheiten, je nach Landesrecht auch der unmittelbaren Bürgerbeteiligung. Damit verbleibt unausweichlich die Unsicherheit, ob ein Gleichlauf von 17 (oder 16) Partnern erreicht werden kann. Besonders missliche Situationen könnten entstehen, wenn sich die Landesregierungen verpflichten, auf einheitliche Regelungen hinzuwirken, die Landesparlamente sich dem aber schlicht verweigern.

\section{Fazit}

Wie auch immer die künftigen Verschuldungsregeln aussehen werden, ob es mit Modifikationen beim Konzept der Art. 109 Abs. 2, Art. 115 Abs. 1 GG bleibt oder aktuelle ökonomische Auffassungen in die Verfassungen und das konkretisierende Gesetzesrecht Eingang finden sollten, eines sollte klar sein: Ein möglichst weitgehender Gleichlauf von bundes- und landesrechtlichen Regeln ist sinnvoll. Halbwegs sicher kann aber eine Übereinstimmung nur erreicht werden, wenn das Grundgesetz als Verfassung nicht nur des Bundes, sondern in diesem Fall auch des Gesamtstaates verbindliche Vorgaben zugleich für den Bund und die Länder enthält. Die Bundesverfassung kann die Verschuldungspolitik der Länder nicht bis in alle Einzelheiten regeln, sie kann sehr wohl aber, wie im geltenden Recht durch Art. 109 Abs. 2 GG, Leitlinien aufstellen. Das berührt die der Verfassungsänderung entzogene Garantie der bundesstaatlichen Struktur und der Gliederung des Bundes in Länder (Art. 20 Abs. 1 i.V.m. Art. 79 Abs. 3 GG) nicht. Bundesverfassungsrechtliche Verschuldungsgrenzen für Bund und Länder betreffen die grundgesetzliche Ausprägung des Bundesstaatsprinzips. Inhaltlich problematisch werden sie erst dann, wenn sie die Länder auf der Einnahmeseite, unter Berücksichtigung aller Möglichkeiten zur Einnahmenbeschaffung, so einschränken, dass eine aufgabengerechte Finanzausstattung der Länder nicht mehr gewährleistet ist. In den Details ist mit Landesregelungen zu rechnen, die vom Bundesrecht abweichen. Wichtiger als das inhaltliche Konzept im Einzelnen wird der Wille der Finanzpolitik sein, den Schuldenabbau einzuleiten und die Neuverschuldung zu stoppen. Beides ist vorrangig eine politische Herausforderung und weniger von den normativen Vorgaben der Kreditlimitierung abhängig. Bezeichnend ist allerdings, dass alle gegenwärtigen Überlegungen der Politik zur zukünftigen rechtlichen Koordinierung der Kreditaufnahme von Bund und Ländern Elemente der Zentralisierung und Vereinheitlichung aufweisen, die mit den ursprünglichen Zielsetzungen der Föderalismusreform, Entflechtung und Stärkung der Eigenverantwortung von Bund und Län- 
dern, keine Berührungspunkte mehr aufweisen. Das aber ist konsequent. Wenn die vertikale Aufgabenverteilung den Ländern wenig Spielraum bei der Gesetzgebung belässt, die vertikalen und horizontalen Verklammerungen beim Finanzausgleich unverändert bleiben sollen, dann muss auch in der Verschuldungspolitik des Bundesstaates die zentral gesteuerte Kooperation aufrechterhalten bleiben. 